\title{
Influence of thermal environments on the growth of bulk cadmium zinc telluride (CZT) single crystals
}

\author{
V. Carcelén ${ }^{\mathrm{a}, *}$, N. Vijayan ${ }^{\mathrm{a}, \mathrm{b}}$, J. Rodríguez-Fernández ${ }^{\mathrm{a}, \mathrm{d}}$, P. Hidalgo ${ }^{\mathrm{c}}$, J. Piqueras ${ }^{\mathrm{c}}$, \\ N.V. Sochinskii ${ }^{\text {d }}$, J.M. Pérez ${ }^{\text {e }}$, E. Diéguez ${ }^{\text {a }}$ \\ ${ }^{\text {a }}$ Laboratorio de Crecimiento de Cristales, Dpto. Física de Materiales, Facultad de Ciencias, University Autónoma de Madrid, 28049 Madrid, Spain \\ ${ }^{\mathrm{b}}$ National Physical Laboratory, New Delhi 110 012, India

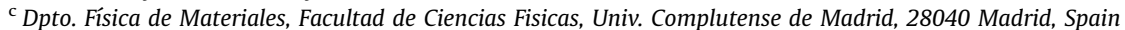 \\ ${ }^{\mathrm{d}}$ Instituto de Microelectronica de Madrid, CNM-CSIC, Polo Technologico de Madrid, Tres Cantos 28760, Madrid, Spain \\ e Laboratorio General de Electronica y Automatica, CIEMAT, Edificio 22, Avenida Complutense 22, E-28040 Madrid, Spain
}

\section{A R T I C L E I N F O}

\section{Article history:}

Received 4 November 2008

Received in revised form

2 January 2009

Accepted 13 January 2009

Communicated by R. James

Available online 21 January 2009

PACS:

68.08.De

81.05.Dz

81.10.Bk

29.40.-n

Keywords:

A1. Characterization

A2. Bridgman technique

A2. Growth from melt

A2. Growth from vapour

B2. semiconducting II-VI materials

\begin{abstract}
A B S T R A C T
The II-VI compound semiconductor crystal cadmium zinc telluride (CZT) is very important in the field of room-temperature radiation detectors and medical imaging applications. In the present study, bulk CZT single crystal has been grown by (i) oscillatory Bridgman technique, (ii) from vapour phase using pyrolytic boron nitride ampoule in the Bridgman geometry, and (iii) by using a Pt tube used for the ampoule support as a cold finger. Several improvements were found in the thermal environments such as the effects of superheating and reduced growth velocity, as well as improvements in the grain size and zinc composition along the ingot. The compositional homogeneity and its current-voltage characteristic behaviour have been analysed using energy dispersive X-ray analysis and $I-V$ method, respectively.
\end{abstract}

(c) 2008 Elsevier B.V. All rights reserved.

\section{Introduction}

II-VI compound semiconductor materials have shown great promise for applications in the field of room temperature $\mathrm{X}$-ray and $\gamma$-ray detectors, security and medical imaging fields [1-3]. Even though various detector materials are available (e.g. $\mathrm{Si}, \mathrm{Ge}, \mathrm{HgI}_{2}$ ) in the market, $\mathrm{Cd}_{x} \mathrm{Zn}_{1-x} \mathrm{Te}(\mathrm{CZT})$ is a leading semiconductor material and getting more attention because of its higher atomic number and density, with the advantage of operating at room temperature [4]. It does not require any cooling apparatus as needed for $\mathrm{Si}$ and Ge based detectors. CZT ingots have already been grown by several researchers using different methods [5-6], but the quality of the as-grown ingots is still a challenge for detector applications. Normally defects

\footnotetext{
*Corresponding author. Tel.: +34 914974784; fax: +34914978579.

E-mail address: veronica.carcelen@uam.es (V. Carcelén).
}

and vacancies are formed during high-temperature crystal growth, and they affect the detector performance. The thermal parameters always play a major role in the solid-liquid interface during the solidification process, and they affect the compositional homogeneity of the material. By keeping all the thermal growth factors in mind, we tested some important parameters, viz. modifying the thermal environments in a Bridgman geometry using a Pt tube as a cold finger in order to reduce the growth velocity and zinc segregation, as well as growth from the vapour phase using the Bridgman geometry with a PBN crucible to allow a special temperature profile for the feed material. Both experiments have the added possibility to perform with in-situ superheating. We have successfully performed the above experiments and confirmed their reproducibility. These methods are inexpensive, but at the same time, the possibility of obtaining detector-grade single crystals is higher than with conventional methods. Details are given in the subsequent sections. 


\section{Experimental}

In the present experimental study, three crystal growth methods have been adopted for the growth of CZT single crystal: (i) growth by oscillatory Bridgman technique (OBR), (ii) growth from the vapour phase (VP) using pyrolitic boron nitride ( $\mathrm{pBN}$ ) crucible and (iii) effect of a Pt cold finger tube (CFT).

In these three growth methods, after performing the preliminary cleaning process of the ampoule, the high-purity raw materials of $\mathrm{Cd}, \mathrm{Zn}$ and Te (zinc: Alfa Aesar 6N; and $\mathrm{Cd}$ and Te: PPM $6 \mathrm{~N}$ pure metals) were charged into the ampoule according to the $\mathrm{Cd}_{0.85} \mathrm{Zn}_{0.15} \mathrm{Te}$ composition, and then the ampoule was pumped to high vacuum $\left(10^{-6}\right.$ mbar $)$ and carefully sealed. The detailed procedure has already been reported [7].

In the first approach, the CZT single crystals were grown by the OBR method following the technique reported by Saucedo et al. [8]. The experimental set up consists of a single-zone furnace, which is able to rotate in clockwise and counter-clockwise directions. It is controlled by home-made software. Eleven thermocouples were circularly placed around the ampoule in order to control the temperature precisely. Then, the whole ampoule assembly was carefully placed into the centre of the Bridgman furnace with mullite support. The temperature was controlled by a Eurotherm temperature controller with an accuracy of $\pm 0.1^{\circ} \mathrm{C}$. The preheating and the crystal growth process have been carried out by setting different temperature ramp rates, which were described in our previous article [7].

In the second approach, the CZT crystal was grown from the vapour phase (VP) using a pBN crucible in the Bridgman geometry. For this VP growth, three individual Kanthal furnaces were fixed with each other in order to get the required temperature profile. The raw materials were charged into the pBN crucible, and then the crucible was inserted in the quartz ampoule leaving the same area as an empty space to account for its vapour pressure. The pBN crucible has the dimensions of 23-mm diameter, $15-\mathrm{cm}$ length and $0.5-\mathrm{mm}$ wall thickness, and it was housed inside a quartz ampoule having the dimensions of $25-\mathrm{mm}$ diameter, $30-\mathrm{cm}$ length and $1.5-\mathrm{mm}$ in thickness. The inserted pBN crucible was held in place by two quartz pins located $6 \mathrm{~cm}$ from the bottom of the quartz ampoule, whereas the top portion of the pBN crucible was supported by two other quartz pins in order to fix the crucible at the centre. Then the whole assembly was sealed under high vacuum. The gap between the pBN crucible and the wall of the quartz tube is $1 \mathrm{~mm}$, in order to allow the passage of vapour of the CZT material which would deposit at the bottom of the quartz ampoule. It is worth mentioning that we have not carried out the graphitization of the quartz ampoule due to non-adhesive nature of the pBN for CZT material. The temperature of the whole furnace was controlled by different thermocouples, which are placed in the bottom as well as circularly placed throughout the ampoule. The temperature of the furnace was fully controlled by separate Eurotherm controllers according to the different temperature ramps. The ampoule temperatures at different positions were recorded using a computer.

In the third approach (CFT), the suitable thermal environments have been created by introducing a Pt tube as the ampoule support; the Pt tube also acted as a cold finger. The Pt CFT was used as the ampoule support, and it was placed at the bottom of the ampoule by adopting the model reported by Kuppurao and Derby [9] in order to enhance the axial heat flow and reduce the radial heat flow and consequently the growth velocity. The Pt tube has the same diameter as the quartz ampoule, having the dimensions of $28-\mathrm{mm}$ diameter, $20-\mathrm{cm}$ length and $1-\mathrm{mm}$ thickness. The bottom tip portion of the quartz ampoule was carefully adjusted accordingly in order to touch the top portion of the platinum cold finger tube, so it will transport the heat from the crystallized materials to the cold part of the Pt tube. In order to know the exact temperature of the ampoule during growth, eleven $\mathrm{Pt} / \mathrm{Pt}-\mathrm{Rh}$ thermocouples were used. Three were kept at the bottom of the ampoule in its central part with a distance of $10 \mathrm{~mm}$, and the remaining eight thermocouples were placed outside of the ampoule in a circular position with a distance of $10-15 \mathrm{~mm}$. The growth process was carried out by following the procedure described in Ref. [7].

The wafers were cut from the as-grown ingot (approx. 2-mm thickness) and polished on both sides using alumina powders (with the grain size of 5, 1 and 0.05 microns). The surfaces were polished using a Buehler Chemomet cloth in order to remove the uneven material. The polished specimens were examined by different characterization techniques in order to check their suitability for device fabrication. The stoichiometric uniformity was assessed by energy dispersive X-ray analysis (EDAX) using a Leica 440 SEM equipped with a Bruker AXS QUANTAX system. Electrical characterization $(I-V)$ was carried out by using a Keithley electrometer (Model 6514).

\section{Results and discussions}

In the OBR method, the furnace oscillated in a clockwise and counter-clockwise direction at about $15^{\circ}$ for $30 \mathrm{~min}$ at a superheating temperature of $15^{\circ} \mathrm{C}$. During this operation the material is more homogeneous due to better mixing in the melt and tellurium inclusions are reduced. Then the ampoule was slowly translated downward at a rate of $0.4 \mathrm{~mm} / \mathrm{h}$ for the growth process, followed by cooling which takes place with the given cooling rates [7]. Then after a span of three to four weeks a good-quality single crystal with a larger grain size has been harvested [7]. OBR technique leads to yield large grain size crystals and at the same time removes the $\mathrm{Te}$ precipitates in the grown ingot as a consequence of the complete homogenization of the raw materials and the reduction of Te precipitates during growth.

While in the OBR approach the temperature environments correspond to a single furnace. In the VP approach three furnaces are used as shown in Fig. 1. In this technique, the furnace geometry was designed in such a way to have three different regions: (i) in the first region ( $\mathrm{R} 1$ ), the temperature profile is nearly constant in order to achieve the proposed superheating; (ii) in the second region (R2), the temperature is drastically decreased to lower value in order to make a controlled evaporation of the source material from the melt, and (iii) the third region (R3) has

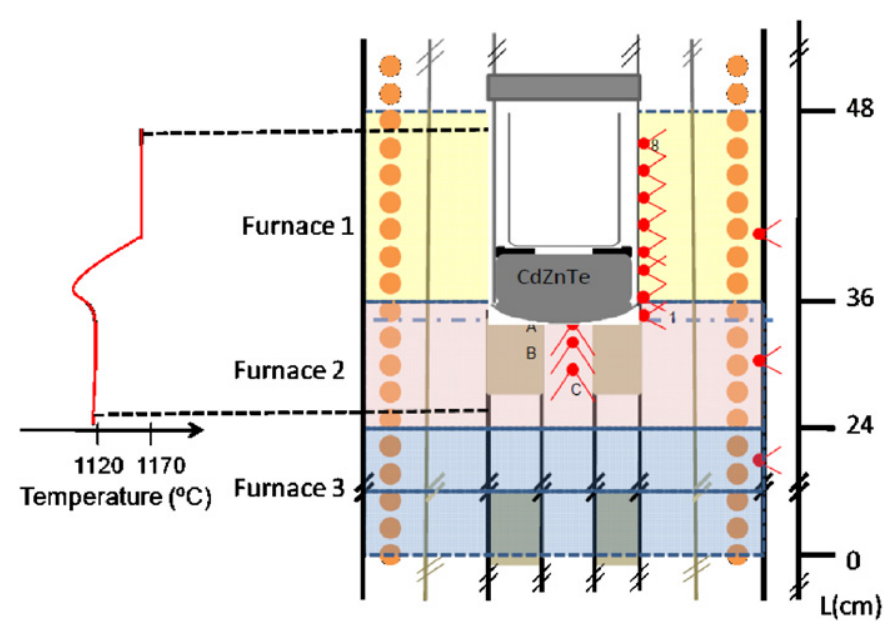

Fig. 1. Schematic representation of the Bridgman furnace used for vapour growth. 
the objective to perform the annealing of the resulted crystal at constant temperature. After achieving the desired temperature profile, the growth experiment was initiated by moving down the quartz tube at a rate of $0.4 \mathrm{~mm} / \mathrm{h}$, the same rate as used in the OBR approach. The vaporization of CZT takes place in the R2 region while the ampoule is going down, and the material condenses and crystallizes in the tip of the ampoule which acts as a nucleation point. Finally, the ampoule is stopped and in-situ annealing is carried out in R3 in order to improve the quality of the grown crystal followed by the cooling process [7]. After successful completion of the experiment, we harvested good-quality CZT crystals. Large, high-quality single crystals [7] were obtained due to the slow growth rate velocity of vapour growth and the annealing step carried out in region R3. A grown bulk CZT single crystal grown by the VP method is shown in Fig. 2 .

In the CFT approach, the Bridgman geometry is used with a single-zone furnace. The thermal environments were modified by the introduction of a Pt tube, in order to modify the heat transfer from the crystallized CZT material to the Pt support. The heat transfer variation is due to the difference in thermal conductivities of the CZT and the Pt tube. It results in drastic changes in the temperature gradient as it is shown in Fig. 3, where the value of the temperature gradient at the interface is obtained from values of the three thermocouples located at the melting point at a given time. In consequence of this geometry, the Pt support causes a change in the thermal environment of the system, which leads to a reduction in the growth rate. The decrease in the rate of growth of CZT decreases the number of grains, and consequently there is remarkable improvement in the ingot's crystalline quality as well as lower zinc segregation during growth. These results can be understood by considering the thermal conductivity of CZT in the molten and solid states ( 0.02 and $0.01 \mathrm{~W} / \mathrm{cm} \mathrm{K}$, respectively), the thermal conductivity of Pt $(0.716 \mathrm{~W} / \mathrm{cm} \mathrm{K})$, and the thermal conductivity of quartz $(0.014 \mathrm{~W} / \mathrm{cm} \mathrm{K})[10]$.

The crystals grown with and without a platinum tube were harvested and subjected for compositional and electrical characterization studies. The zinc composition has been analyzed in a

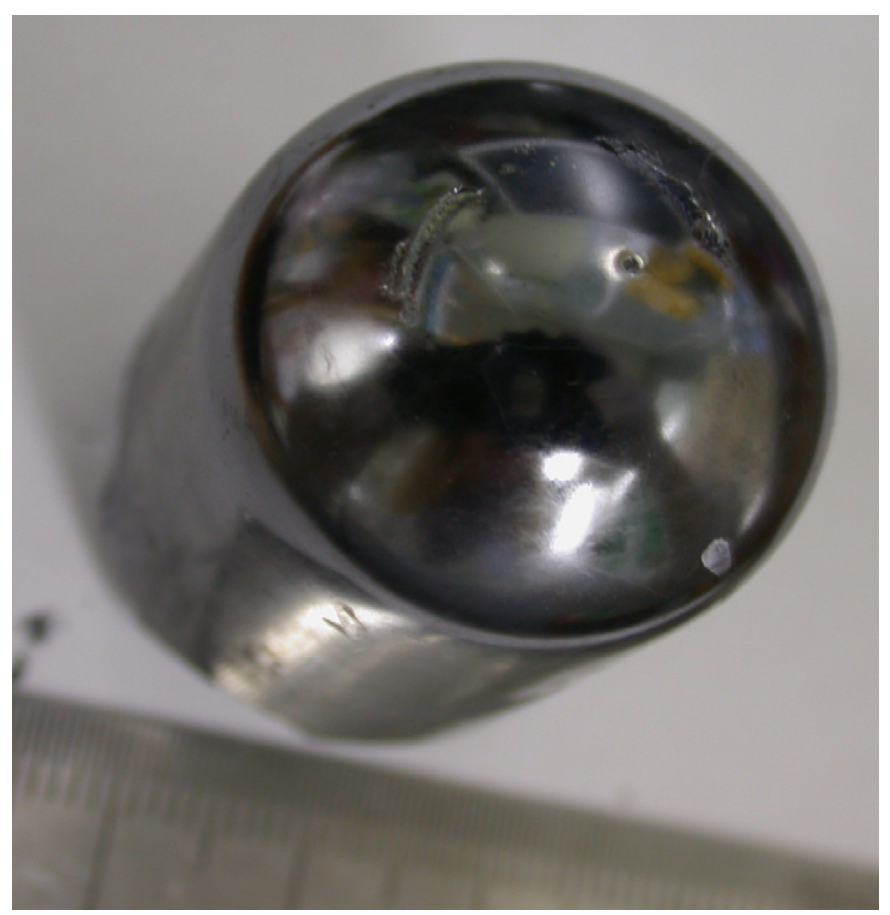

Fig. 2. Single crystal of CZT grown by vapour phase.

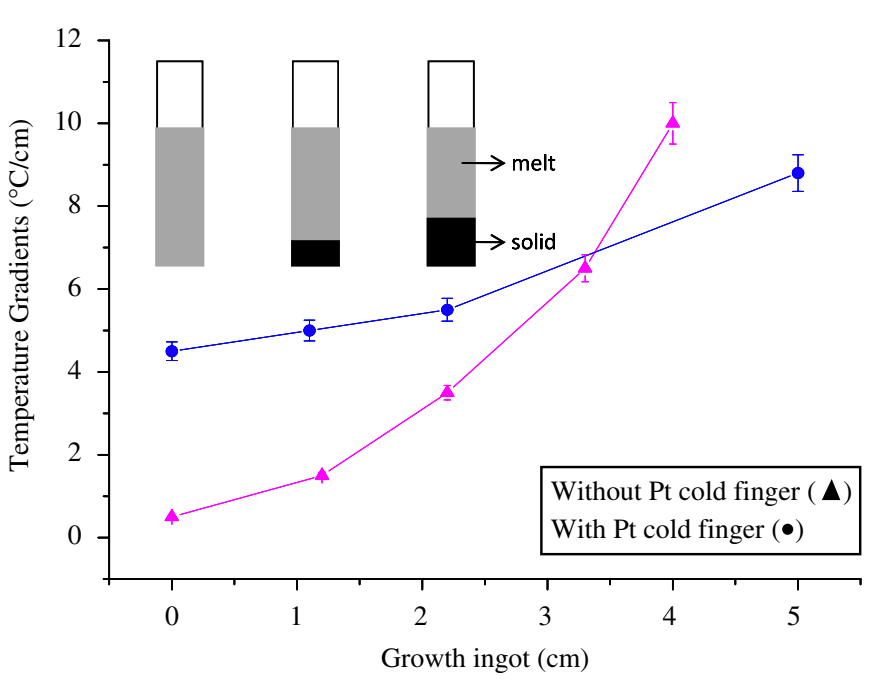

Fig. 3. Temperature gradient of the grown ingot with and without the Pt tube.

$\mathrm{Cd}_{0.85} \mathrm{Zn}_{0.15}$ Te crystal. The results are given in Fig. 4 for samples grown without (a) and with Pt tube (b), which are located along the growth axis at $8 \mathrm{~mm}(\mathrm{a} 1, \mathrm{~b} 1)$ and $25 \mathrm{~mm}(\mathrm{a} 2, \mathrm{~b} 2)$ from the tip of the ampoule. From these figures one can see that the zinc concentration is more uniform for the samples grown with the platinum tube. Non-uniform and lower zinc concentration was observed for the specimens which were grown without platinum tube. From these observations we conclude that the platinum tube used as an ampoule support improves the zinc concentration along the growth axis, whereas the $\mathrm{Zn}$ concentration behaviour in the growth process without Pt support follows the general rule for the segregation coefficient of $\mathrm{Zn}$.

The current-voltage characteristics have been examined by an $I-V$ method at room temperature for the samples grown with and without the Pt tube. This is an important characteristic tool to know the suitability of the material for device fabrication. The polished specimens were coated with gold for contacts. The measurements have been carried out at different positions in the same crystal. The results are shown in Table 1. Although all the values are in same order of magnitude, one observes an increase in the absolute value along the growth axis. More work relevant to these studies is in progress.

From the foregoing discussions it can be concluded that the CZT single crystals have been successfully grown by OBR and vapour phase methods. The introduction of the platinum cold finger tube enhances the grain size of the as-grown ingot, reduces the growth velocity, and at the same time, reduces the zinc segregation which is nearly constant throughout the whole ingot. The Pt tube will be an added advantage for the growth of large CZT crystals for industrial applications.

\section{Conclusions}

The quality of CZT single crystal has been improved by modifying the thermal environments of the growth process. The growth of CZT by vapour phase with the Bridgman geometry using PBN crucible shows a good crystalline quality, which will be useful for industrial purposes. The insertion of a Pt cold finger tube in the bottom of the quartz ampoule drastically changes the thermal environments and reduces the growth velocity. As a consequence, it enhances the growth rate and reduces the zinc segregation. The current voltage $(I-V)$ characteristics have been evaluated by an $I-V$ method and found that there are no remarkable changes in the resistivity for the crystals grown by both methods. 

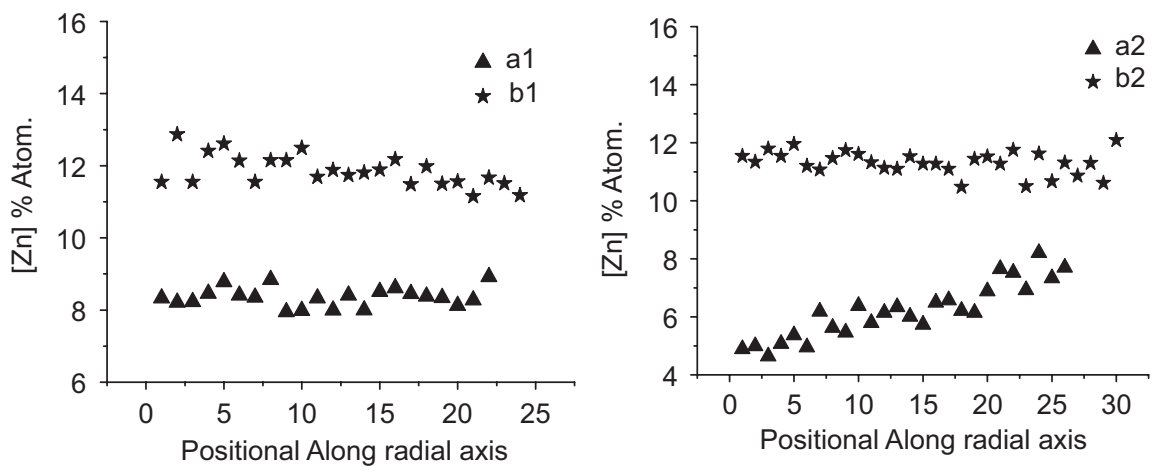

Fig. 4. Grown samples analysed by EDAX (with and without the Pt tube).

Table 1

Resistivity values of the samples grown with and without the Pt tube support.

\begin{tabular}{llll}
\hline Growth & $\begin{array}{l}\text { Position of } \\
\text { sample }\end{array}$ & $($ Zn $) \%$ at & $\begin{array}{l}\text { Resistivity } \\
(\Omega \mathrm{cm}) \times 10^{8}\end{array}$ \\
\hline Without Pt & $11 / 14$ & 13 & 5.1 \\
& $26 / 29$ & 15 & 7 \\
& $45 / 48$ & 8 & 8.5 \\
With Pt & & & \\
& $11 / 14$ & 11.3 & 2.4 \\
& $26 / 29$ & 11.8 & 6.5 \\
\hline
\end{tabular}

\section{Acknowledgements}

This work was partially supported by the following Projects: ESP2006-09935, Spanish "Ministerio de Educación y Ciencia"; S-0505/MAT-0279, Spanish "Comunidad de Madrid"; FP7-SEC2007-01, European Commission, and Contract number 14240/00/ $\mathrm{NL} / \mathrm{SH}$, European Space Agency. One of the authors VC is thankful to the Ministry of Education and Science, Spain for the financial support. The author NV is grateful to Department of Science and Technology, Govt. of India for providing the BOYSCAST fellowship. The author JRF is thankful to the Universidad Autónoma of Madrid for financial support.

\section{References}

[1] G. Li, W. Jie, H. Hua, Z. Gu, Prog. Cryst. Growth Charact. Mat. 46 (2003) 85.

[2] A.E. Bolotnikov, G.S. Camarda, G.A. Carini, Y. Cui, L. Li, R.B. James, Nucl. Inst. Meth. Phys. Res. A 579 (2007) 129.

[3] M.J. Harrison, A. Kargar, D.S. McGregor, Nucl. Inst. Meth. Phys. Res. A 579 (2007) 134

[4] M.C. Duff, D.B. Hunter, A. Burger, M. Groza, V. Buliga, D.R. Black, Appl. Surf. Sci. 254 (2008) 2889.

[5] I. Jung, H. Krawczynski, A. Burger, M. Guo, M. Groza, Astropart. Phys. 28 (2007) 397.

[6] M. Schieber, R.B. James, H. Hermon, A. Vilensky, I. Baydjanov, M. Goorsky T. Lam, E. Meerson, H.W. Yao, J. Erickson, E. Cross, A. Burger, J.O. Ndap G. Wright, M. Fiederle, J. Cryst. Growth 231 (2001) 235.

[7] V. Carcelen, N. Vijayan, E. Dieguez, A. Zappetini, M. Zha, S. Lamine, A. Fauler M. Fierderle, J. Optoelect. Adv. Mat. 10 (2008) 3135.

[8] E. Saucedo, P. Rudolph, E. Dieguez, J. Cryst. Growth 310 (2008) 2067.

[9] S. Kuppurao, J.J. Derby, J. Cryst. Growth 172 (1997) 350.

[10] V. Carcelen, E. Dieguez, Patent demand No. 200801503, Spain. 